\title{
Dynamic structural changes in a single catalyst particle during single walled carbon nanotube growth
}

Pin Ann Lin ${ }^{1,2}$, Zahra Hussaini ${ }^{1}$, Juan C Burgos Beltran ${ }^{3}$, Jose Leonardo Gomez Ballesteros ${ }^{3}$, Perla B. Balbuena $^{3}$, Renu Sharma ${ }^{1}$

1. Center for Nanoscale Science and Technology, National Institute of Standards and Technology, Gaithersburg, MD, USA

2. NanoCenter of Maryland, University of Maryland, College Park, MD, USA

3. Department of Chemical Engineering, Texas A\&M University, College Station, TX, USA

The interaction of gases with a solid catalyst nanoparticle during catalysis is a non-equilibrium process. For example, during single-walled carbon nanotube (SWCNT) growth, carbon atoms diffuse in and out of the catalyst particle, causing variations in the chemical potential, and possibly structure, of the particle. We have employed an environmental scanning transmission electron microscope (ESTEM), with an aberration corrector, operated at $300 \mathrm{kV}$ to record real-time, atomic-resolution videos (6 frames $\mathrm{s}^{-1}$ ) with $\approx 1000$ frames of SWCNT growth from a Co-Mo/MgO system in a $\mathrm{C}_{2} \mathrm{H}_{2}$ gaseous environment at synthesis temperatures. The time-resolved videos, generating large data sets, are used to identify individual reaction steps. We have developed methods to analyze these large data sets accurately and efficiently.

The simultaneous existence of two regions having different structures (pure Co and Co-carbide) within an individual particle during SWCNT growth (Fig. 1a) was detected using fast Fourier transform (FFT) analysis of the catalyst particle images. In order to determine the catalyst particle's structure over time, an image processing scheme that utilizes a combination of home-built and publicly-available algorithms for image drift correction, noise reduction, and template matching to accurately locate the position of atomic columns has been created. For each position, the distances between neighboring atom columns were averaged, giving a local spacing value which was used to determine whether a local region was Co or Co-carbide (Fig. 1b). Figure 2a shows the fluctuation in Co volume fraction, which suggests that the catalyst particle has a changing stoichiometry as a function of time. In addition to the particle structure measurements, the growth length of the SWCNT was measured (Fig. 2b). We found that when the Co fraction increases - carbon output is greater than carbon intake - the tube growth rate increases. Similarly, when the Co fraction decreases - carbon intake is greater than carbon output - the tube growth rate decreases.

The fluctuation of carbon content inside the catalyst particle can be directly related to the nanotube growth, which can then be used to calculate rates for reaction steps, such as bulk or surface diffusion of carbon atoms through a particle and their incorporation into a nanotube. A detailed description of structural analysis approach and quantitative measurements of catalyst carbon content and nanotube growth will be presented. 

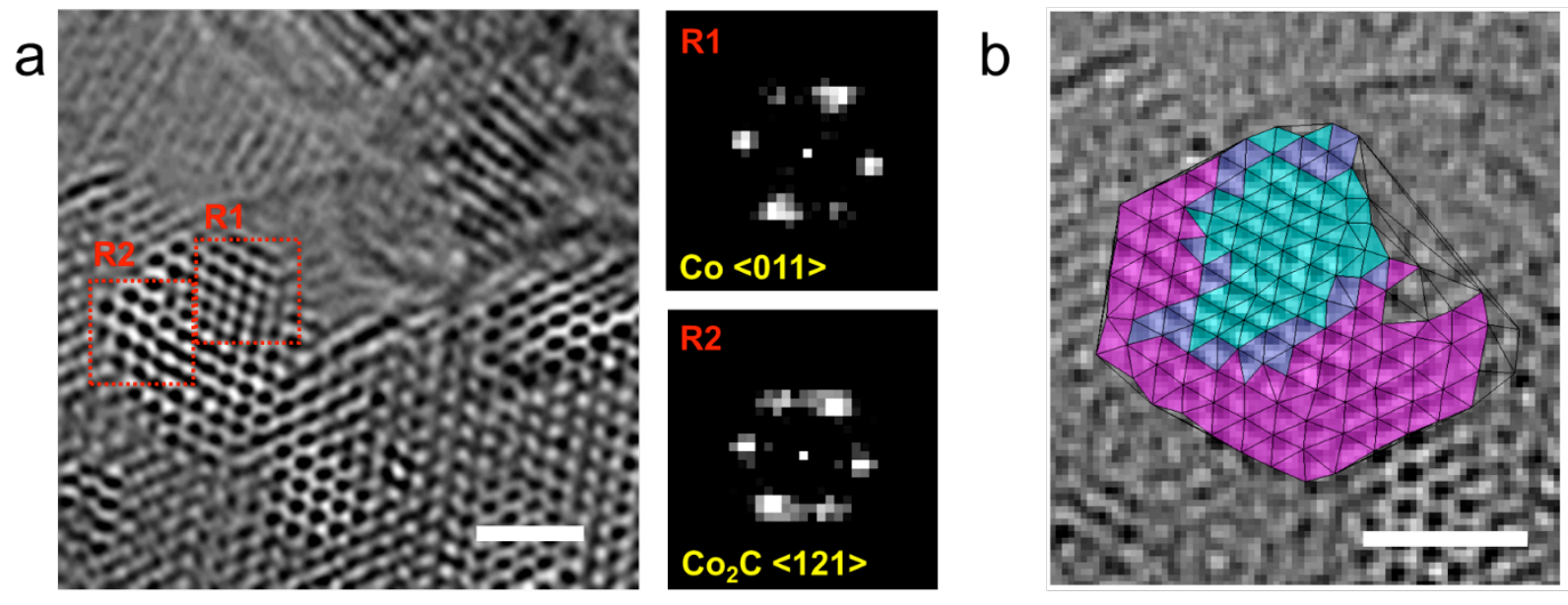

Figure 1. (a) A high resolution image of SWCNT growth from a catalyst particle that contains two different structures $\left(\mathrm{Co}\right.$ and $\left.\mathrm{Co}_{2} \mathrm{C}\right)$, which are identified by FFT images of $\mathrm{R} 1$ and $\mathrm{R} 2$ regions. (b) Blue and violet areas are marked for Co and Co-carbide, respectively, and an intermediate color in between shows the boundaries. Scale bars are $1 \mathrm{~nm}$.
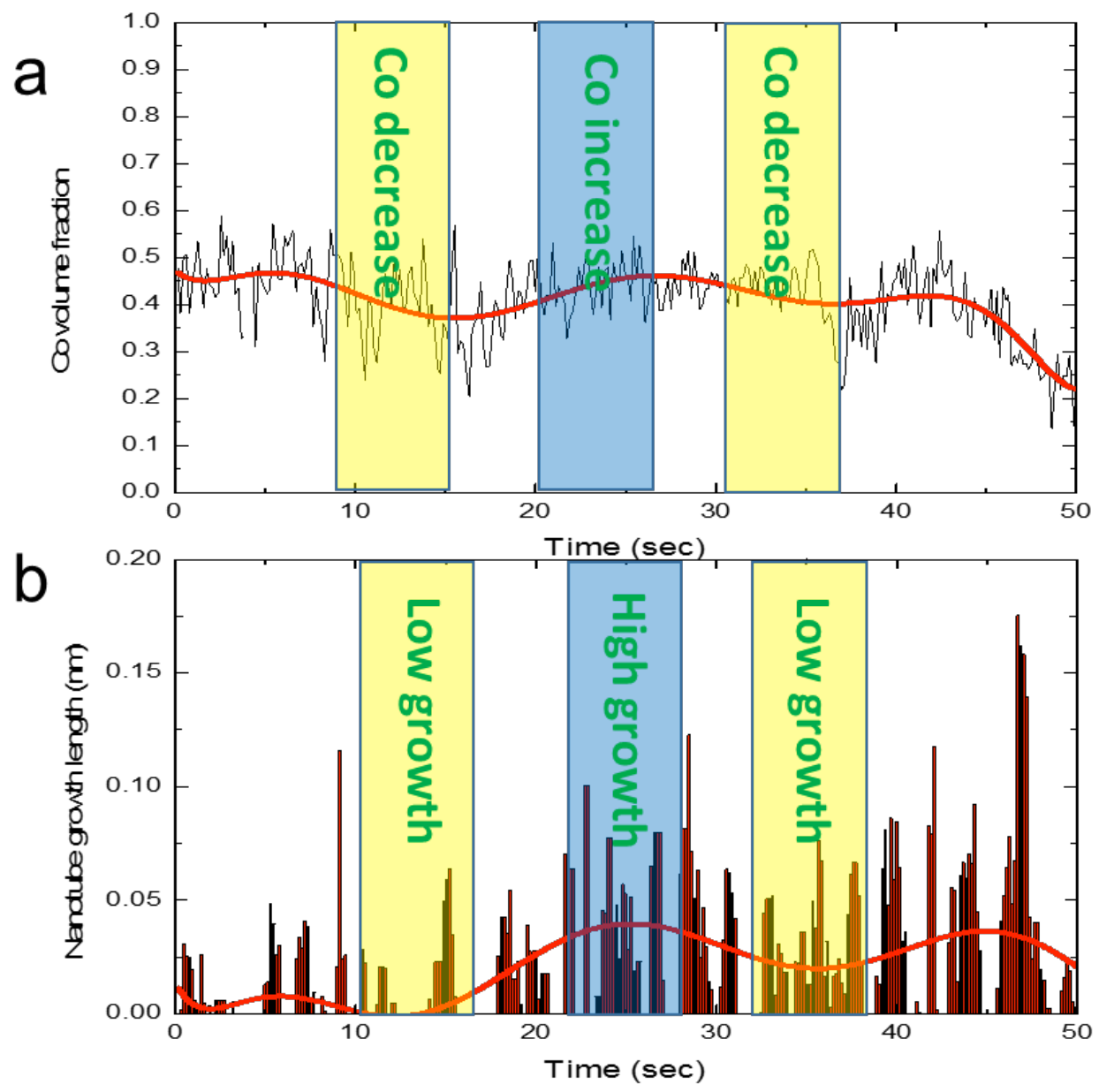

Figure 2. Fluctuations in (a) Co volume fraction and (b) nanotube growth length as a function of time. 